\title{
Microwave Field Strength Computing for the Resonator Designs and Filters
}

\author{
M. RadmiloviĆ-Radjenović* ${ }^{*}$ B. Radjenović And M. SAViĆ \\ Institute of Physics, University of Belgrade, Pregrevica 118, 11080 Zemun, Serbia
}

(Received March 29, 2013)

\begin{abstract}
Recent development of microwave pulse generators, which are now capable of delivering very short and very intensive pulses requires properly generalized classical breakdown theory. On the other hand, the trend to design microwave devices as small and compact as possible, leads to a concern about the concomitant breakdown strength of the construction, involving more complicated geometries, such as in microwave resonators and filters. In this paper, several aspects of microwave breakdown field strength in commercially available resonator designs and filters are presented and analyzed. The numerical predictions based on the Slater theorem are compared with the analytical results and predictions of the fluid approach, demonstrating very good agreement.
\end{abstract}

DOI: 10.12693/APhysPolA.129.289

PACS/topics: 52.80.-s, 52.90.+z

\section{Introduction}

Microwave breakdown in gases under various physical and technical conditions is well-known and intensively studied problem from early days of gaseous electronics [1-3]. More recent, interest in microwave-induced breakdown is shifted to studies of microwave breakdown at atmospheric pressures due to its relevance both for industrial applications [4-6] and for a deeper understanding of fundamental plasma behavior [7-9]. The microwave breakdown strength of the gas is useful information for high power filter and antenna engineering [10], for determination of the conductivity and dielectric constant of a plasma [11], for modification the ionosphere for long range radio propagation [12], etc. The microwave breakdown phenomenon provides both problems and opportunities reflected in the applications of the microwave breakdown theory. The application can be divided into two main categories: the so-called no-breakdown devices - where breakdown should be avoided for proper operation of the device and breakdown devices - where breakdown is an intrinsic feature of operation.

In numerous microwave devices, the geometrical configuration contributes to local strongly enhanced microwave fields. Such regions are potentially dangerous from the point-of-view of breakdown since the field here may be much stronger than the globally predicted breakdown field. On the other hand, a locally overcritical field does not necessarily imply global breakdown. In that case, the influence of local field enhancement on the global breakdown condition has to be included.

This paper contains result of our studies of the microwave induced dielectric breakdown of gases in a uniform electric field. Cavity resonators have often been used $[13,14]$, but field distributions are inhomogeneous,

*corresponding author; e-mail: marija@ipb.ac.rs resulting in a spatially dependent ionization rate and breakdown which is strongly influenced by the diffusion of charged particles out of the high electric field region.

\section{Models}

Several different models of the microwave breakdown criteria exist, with varying degree of accuracy in the description. Fluid models provide description with enough details that can be used in large classes of research becoming very important for technical applications [15]. On the other hand, the kinetic approach has potential to offer a very detailed description of the mechanism and represents the basis on which other models rely. The great details in descriptions, however, make the kinetic approach very complex and more useful in pure physical research with simplified geometry.

\subsection{The Slater theorem for computing the electric field in the gap}

The electric field inside a microwave resonator can be determined analytically based on an analytic solution to the Maxwell equations. The electric and magnetic fields inside a resonator have a complicated spatial variation, which nevertheless satisfies the Helmholtz wave equation under the constraints of the conductive boundary conditions. Analytic solutions of the electric and the magnetic fields exist only for a few simple cavity geometries. It is not usually possible to analytically find the magnitude of the fields inside most resonators, let alone their geometric distribution. The field strength can be determined numerically using full-wave electromagnetic field simulation [14].

The perturbation theory of Slater is used here to obtain the local electric field. Only a small fraction of the empty space inside an electromagnetic resonator is filled with the dielectric material with relative permittivity $\varepsilon_{\mathrm{r}}$, and relative permeability $\mu_{\mathrm{r}}$. The interaction between the magnetic permeability of the material and the magnetic field of the resonator will cause the reactance to increase 
and thus raise the resonant frequency by the inductive loading. Likewise, the interaction between the electric permittivity of the material and the electric field of the resonator will cause the reactance to decrease and thus lower the resonant frequency by the capacitive loading. This frequency shift, $f_{0}-f$ can be expressed via the Slater theorem [14]:

$$
\begin{gathered}
\omega^{2}=(2 \pi f)^{2}= \\
\omega_{0}^{2}\left[1+\frac{\int_{\text {dielectric }}\left(\mu_{0} \mu_{\mathrm{r}} H^{2}-\varepsilon_{0} \varepsilon_{\mathrm{r}} E^{2}\right) \mathrm{d} V}{\int_{\text {cavity }}\left(\frac{1}{2} \mu_{0} H^{2}+\frac{1}{2} \varepsilon_{0} E^{2}\right) \mathrm{d} V}\right],
\end{gathered}
$$

where $H$ and $E$ represent the magnetic and electric fields, respectively, while $\omega_{0}=2 \pi f_{0}$ is the original, unperturbed angular frequency. The top integral is evaluated over the dielectric perturbation, and gives the energy stored in the perturbation. The bottom integral is evaluated over the entire empty cavity and is equal to the peak energy stored per cycle in the resonator $-U_{0}$.

\subsection{An analytical-experimental approach}

There is also a simple complementary approach obtained by combination of an analytical and experimental investigations suggested in [13]. Actually, the method is based on experimental data of the breakdown voltages at low pressures and their extrapolation over a wide range of pressures. The expression for the breakdown voltage is written in the form [13]:

$$
E_{\mathrm{b}}=3.75 p\left[1+\left(\frac{2 \pi f}{\nu_{\mathrm{c}}}\right)^{2}\right]^{1 / 2}\left(\frac{D}{p L_{\mathrm{D}}^{2}}+6.4 \times 10^{4}\right)^{3 / 16},
$$

where $E_{\mathrm{b}}$ represents the real rms electric field and the characteristic length $L_{\mathrm{D}}$ determines the curvature of the breakdown voltage curve curve.

\subsection{Fluid approach}

In the case of microwave electric fields, the continuity equation describes the time evolution of the electron density $n_{\mathrm{e}}$ which is

$$
\frac{\partial n_{\mathrm{e}}}{\partial t}=\nabla\left(D \mathrm{e} \nabla n_{\mathrm{e}}\right)+\nu n_{\mathrm{e}}
$$

where $D_{\mathrm{e}}$ is the electron diffusion coefficient and $\nu$ is the net production rate of electrons per electron. The microwave breakdown can be determined as a balance between the ionization rate and the loss rate of electrons by diffusion mathematically formulated by relation [15]:

$$
\nu_{i}=\frac{D_{\mathrm{e}}}{\Lambda^{2}},
$$

with the characteristic diffusion length $\Lambda$ depending on the geometry of the discharge vessel, i.e. radius $R$ and height $L$ in accordance with the expression

$$
\frac{1}{\Lambda^{2}}=\left(\frac{2.4}{R}\right)^{2}+\left(\frac{\pi}{L}\right)^{2},
$$

where the diffusion to the end plates is given by the first on the right, while the second term describes the diffusion to the cylindrical walls.
In general, the electron diffusion is anisotropic, but at very high frequencies the transverse and longitudinal diffusions become almost isotropic with the values nearly equal to the transverse one $D_{\tau}$ at $\omega \rightarrow 0$. Therefore, in Eq. (3), the electron diffusion coefficient $D_{\mathrm{e}}$ can be replaced by the transverse diffusion coefficient $D_{\tau}$ that corresponds to the dc field. Combining Eq. (3) with $\nu_{i}=\alpha \mu E$ :

$$
\frac{\varepsilon}{\Lambda^{2}}=\frac{\alpha E_{\mathrm{r}}}{\sqrt{1+\left(\frac{\omega}{\nu_{\mathrm{c}}}\right)^{2}}},
$$

where $\varepsilon=D_{\tau} / \mu$ represents the characteristic energy, while $\mu$ is the electron mobility. When an ac electric field $E_{\mathrm{r}} \cos \omega t$ is applied in a weakly ionized gas, a dominant collision processes are electron-neutral collisions characterized by the collision frequency $\nu_{\mathrm{c}}$. The frequency effect can be comprehended by introducing the effective field strength

$$
E_{\text {eff }}=\frac{\nu_{\mathrm{c}}}{\sqrt{\nu_{\mathrm{c}}^{2}+\omega^{2}}} E_{\mathrm{r}}
$$

Finally, substituting expression for the first Townsend coefficient $\alpha=A p \exp \left(B p / E_{\mathrm{dc}}\right)$ [15] and replacing $E_{\mathrm{dc}}$ by $E_{\text {eff }}$ given by Eq. (6), we obtain expression for the electric field $E_{\mathrm{r}}$ as a function of the pressure $p$ :

$$
E_{\mathrm{r}}=\frac{\varepsilon \sqrt{\omega^{2}+\nu_{\mathrm{c}}^{2}} \exp \left(B p \sqrt{\omega^{2}+\nu_{\mathrm{c}}^{2}} /\left(\nu_{\mathrm{c}} E_{\mathrm{r}}\right)\right)}{\nu_{\mathrm{c}} \Lambda^{2} A p},
$$

where $\omega$ represents angular frequency of the applied field.

\section{Results}

Atmospheric plasma source based on a microstrip splitring resonator (MSSR) is depicted in Fig. 1 with a schematic view given in Fig. 2. We have modelled the

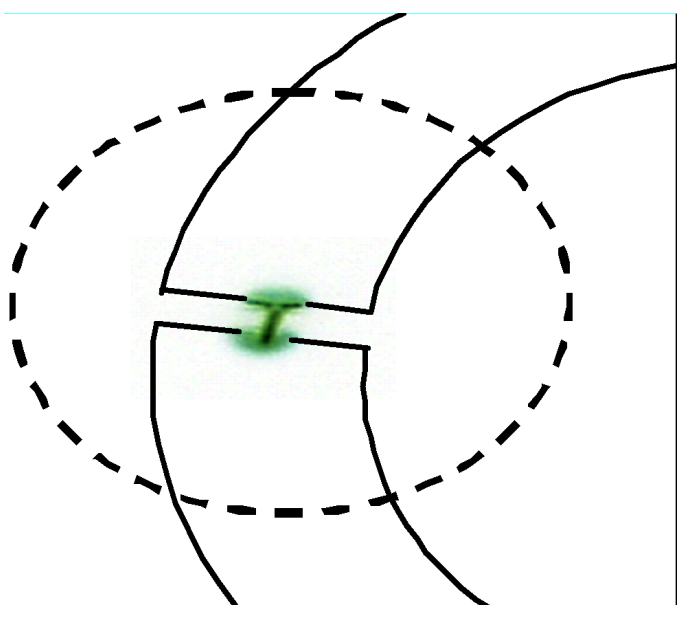

Fig. 1. Microstrip split-ring resonator.

electric field inside a microwave resonator numerically based on evaluation integrals in the Slater theorem. Coupling between electric and magnetic fields inside an electromagnetic resonator is demonstrated in Fig. 3. For the microwave threshold field of the gas which fills the resonator volume, however, the magnetic field is very low 


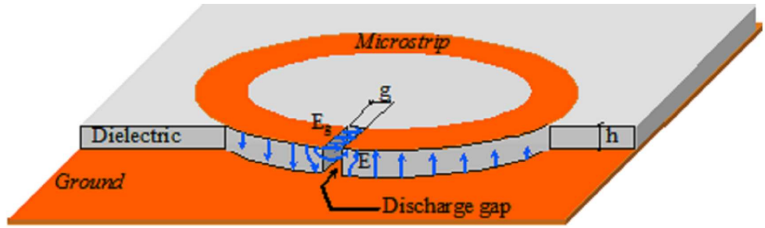

Fig. 2. Schematic view of microstrip split-ring resonator.

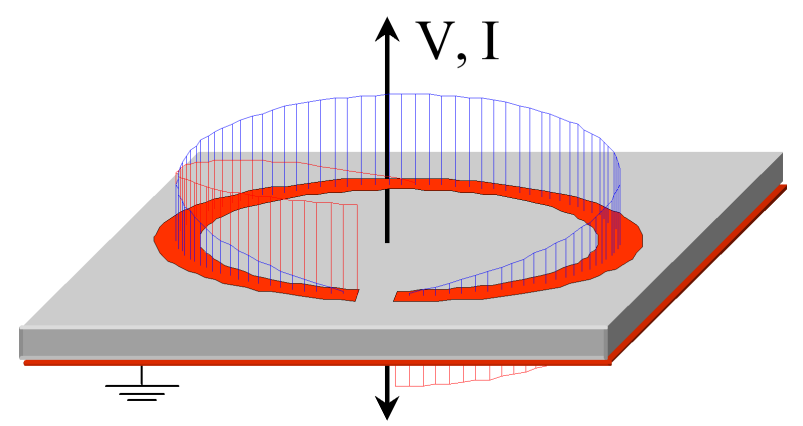

Fig. 3. Schematic view of split-ring resonator.

and can be neglected. Electric field can be treated as uniform over the volume of the dielectric. Calculated values of the magnitude of the electric field are shown in Fig. 4.

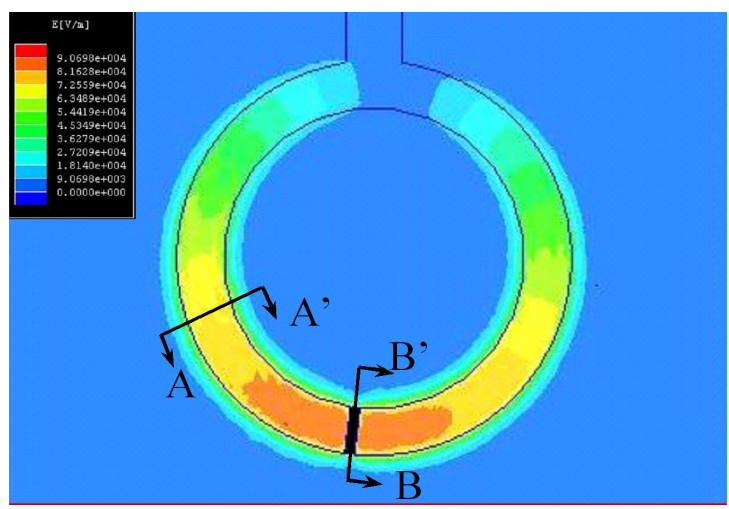

Fig. 4. Magnitude of the electric field.

The breakdown field strength as a function of pressure in nitrogen at microwave frequency of $1.85 \mathrm{GHz}$ is shown in Fig. 5. Our numerical results are presented by solid line, while dot and dash lines correspond to the predictions obtained by using Eqs. (2) and (8), respectively. Theoretical predictions based on both analytical and fluid approaches provide relatively good results at lower pressures. Our numerical results, however, are in a good agreement with the data taken from [14] at all pressure values.

A good agreement between our numerical results (solid line) and results published in [14] (solid symbols) is also achieved for the pressure dependence of the breakdown field strength in nitrogen at $2.76 \mathrm{GHz}$ at the temperature

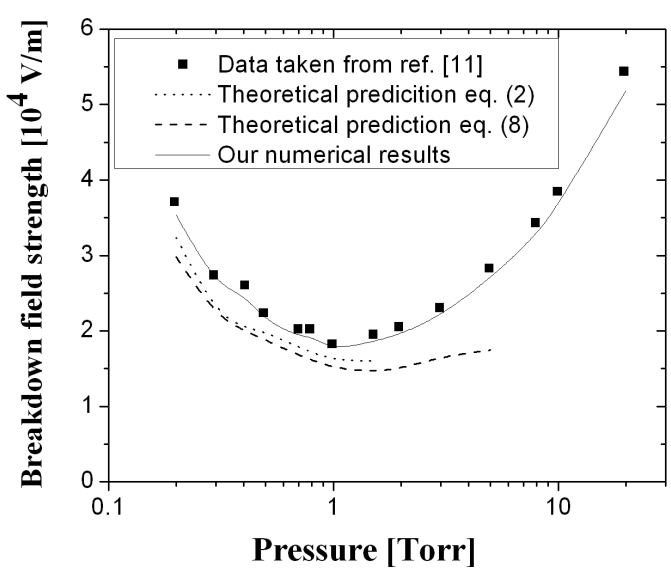

Fig. 5. The breakdown field strength versus the pressure in nitrogen at frequency of $1.85 \mathrm{GHz}$.

of $230 \mathrm{~K}$ (as can be seen from Fig. 6). Finally, how the pressure at the minimum breakdown voltage curve depends on frequency is illustrated in Fig. 7. As expected, there is a linear dependence indicating that the pressure at the minimum increases as the frequency is increased.

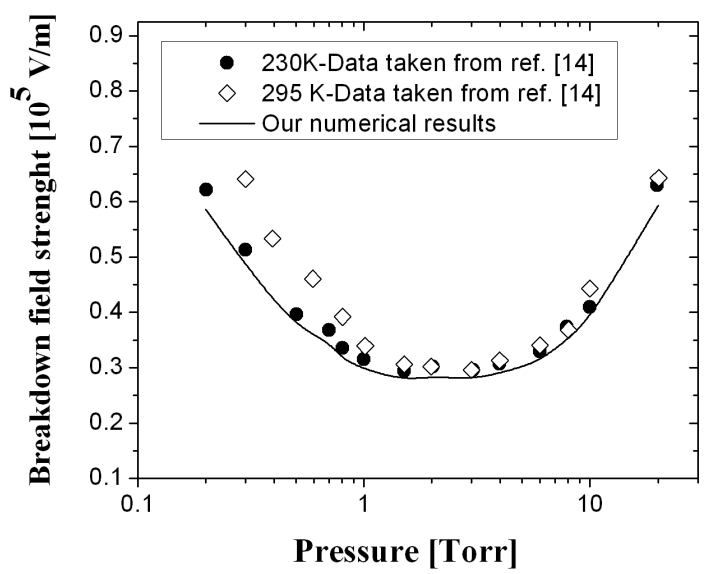

Fig. 6. The pressure dependence of the breakdown field strength in nitrogen at frequency of $2.76 \mathrm{GHz}$.

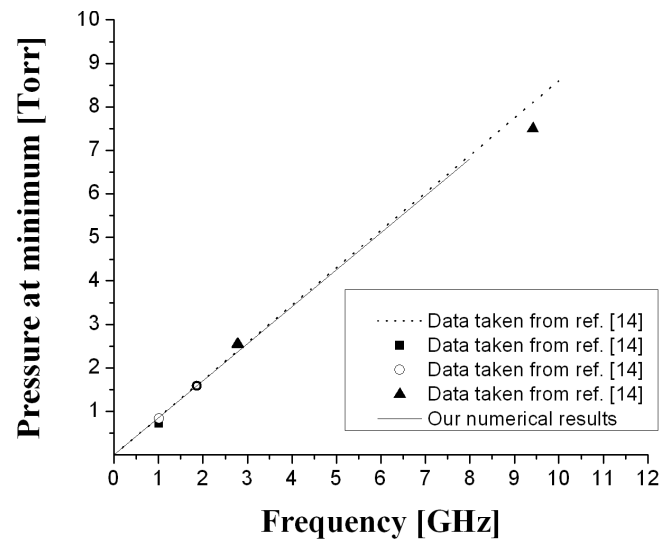

Fig. 7. The pressure at the minimum as a function of frequency in nitrogen. 


\section{Conclusion}

Nowadays, there is a growing interest in microplasma sources due to their economics, portability, less operation costs, and small sizes. The utility derived from macroscale plasmas combined with current microfabrication techniques has allowed for the development of microscale devices which use microdischarges for operation. Having in mind that relatively complicated geometrical configurations in microwave devices enhance the strong local microwave fields and such fields may be much stronger than the globally predicted breakdown field. This paper is devoted to our numerical studies of the microwave field strength computing in commercially available resonator designs and filters. Numerical method is based on Slater's theorem. Our numerical results satisfactorily agree with the available data [14] indicating their applicability for a wide range of parameters.

The results presented here, except for resonator and filters, can be also useful in localized diagnostics of ICs during their manufacture, in choosing appropriate conditions for electro mechanical micro systems which may eventually lead to nanomachining, in localized treatment of materials and assembly of nanostructures and in micro and nanobiological processing and diagnostics.

\section{Acknowledgments}

The work has also been carried out under by Ministry of Education and Science Republic of Serbia O171037 and III41011 projects.

\section{References}

[1] M.J. Druyvestayn, F.M. Penning, Rev. Mod. Phys. 12, 87 (1940).

[2] D.Q. Posin, Phys. Rev. 73, 496 (1948).

[3] S. Krasik, D. Alpert, A.O. McCoubreyn, Phys. Rev. 76, 722 (1949).

[4] F.C. Fehsenfeld, K.M. Evenson, H.P. Broida, Rev. Sci. Instrum. 36, 294 (1965).

[5] M. Radmilović-Radjenović, B. Radjenović, Central Europ. J. Phys. 9, 265 (2011).

[6] B. Radjenović, M. Milanović, M. RadmilovićRadjenović, Phys. Scr. T 149, 014026 (2012).

[7] W.P. Allis, S.C. Brown, Phys. Rev. 87, 419 (1952).

[8] H.J. Oskam, J. Appl. Phys. 27, 848 (1956).

[9] A.D. MacDonald, D.U. Gaskell, H.N. Gitterman, Phys. Rev. 130, 1841 (1963).

[10] F. Werner, D. Korzec, J. Engemann, Plasma Sources Sci. Technol. 3, 473 (1994).

[11] T. Fleisch, Y. Kabouzi, J. Pollack, E. CastañosMartínez, H. Nowakowska, M. Moisan, Plasma Sourc. Sci. Technol. 16, 173 (2007).

[12] D. Anderson, U. Jordan, M. Lisak, T. Olsson, M. Ahlander, IEEE Trans. Microwave Theory Techn. 47, 2547 (1999).

[13] S.K. Remillard, A. Hardaway, B. Jork, J. Gilliland, J. Gibs, Progr. Electromagn. Res. B 15, 175 (2009).

[14] M. Radmilovic-Radjenovic, J.K. Lee, F. Iza and G.Y. Park, J. Phys. D: Appl. Phys 38, 950 (2005).

[15] R. Tomala, U. Jordan, D. Anderson, M. Lisak, Contr. Plasma Phys. 46, 287 (2006). 